\title{
Production and Characterization of a new
}

\author{
Copper(II) Propanoate-Isonicotinamide
}

Adduct obtained via Slow Evaporation and

using Supercritical $\mathrm{CO}_{2}$ as an Antisolvent

Isaac A. Cuadra, Francisco J. Martínez-Casado*, José A. R. Cheda, M. I. Redondo, Concepción Pando, Albertina Cabañas*

Dpto. Química Física, Universidad Complutense, E-28040 Madrid, Spain

*fmarcas@gmail.com,*a.cabanas@quim.ucm.es 


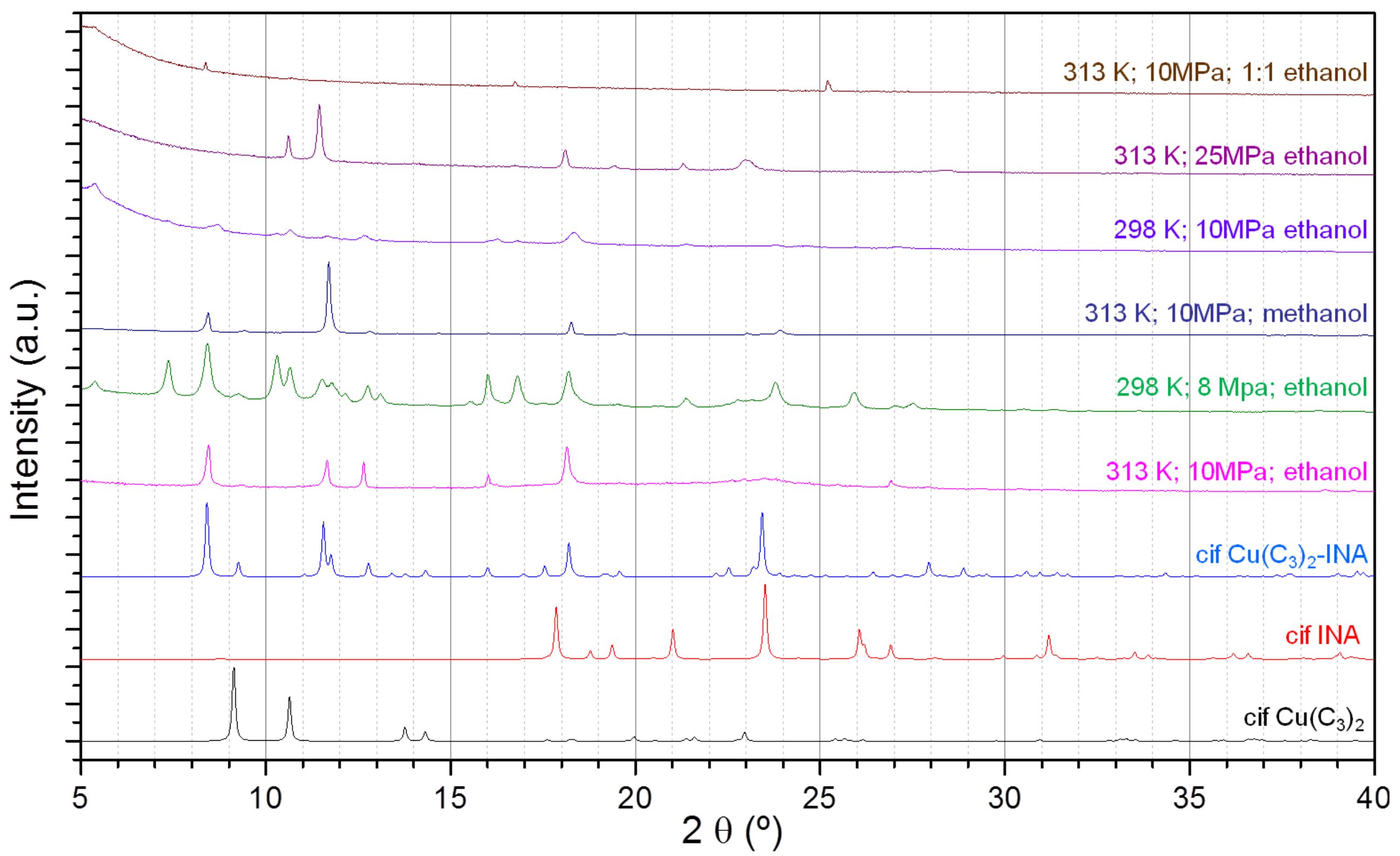

Figure S1. PXRD patterns of the samples precipitated by SAS at the different conditions with a 1:2 $\mathrm{Cu}\left(\mathrm{C}_{3}\right)_{2}$ to $\mathrm{INA}$ molar ratio unless otherwise specified and comparison with the CIF files for $\mathrm{Cu}\left(\mathrm{C}_{3}\right)_{2}$, INA and the $\mathrm{Cu}_{2}\left(\mathrm{C}_{3}\right)_{4}(\mathrm{INA})_{4}$ adduct. 

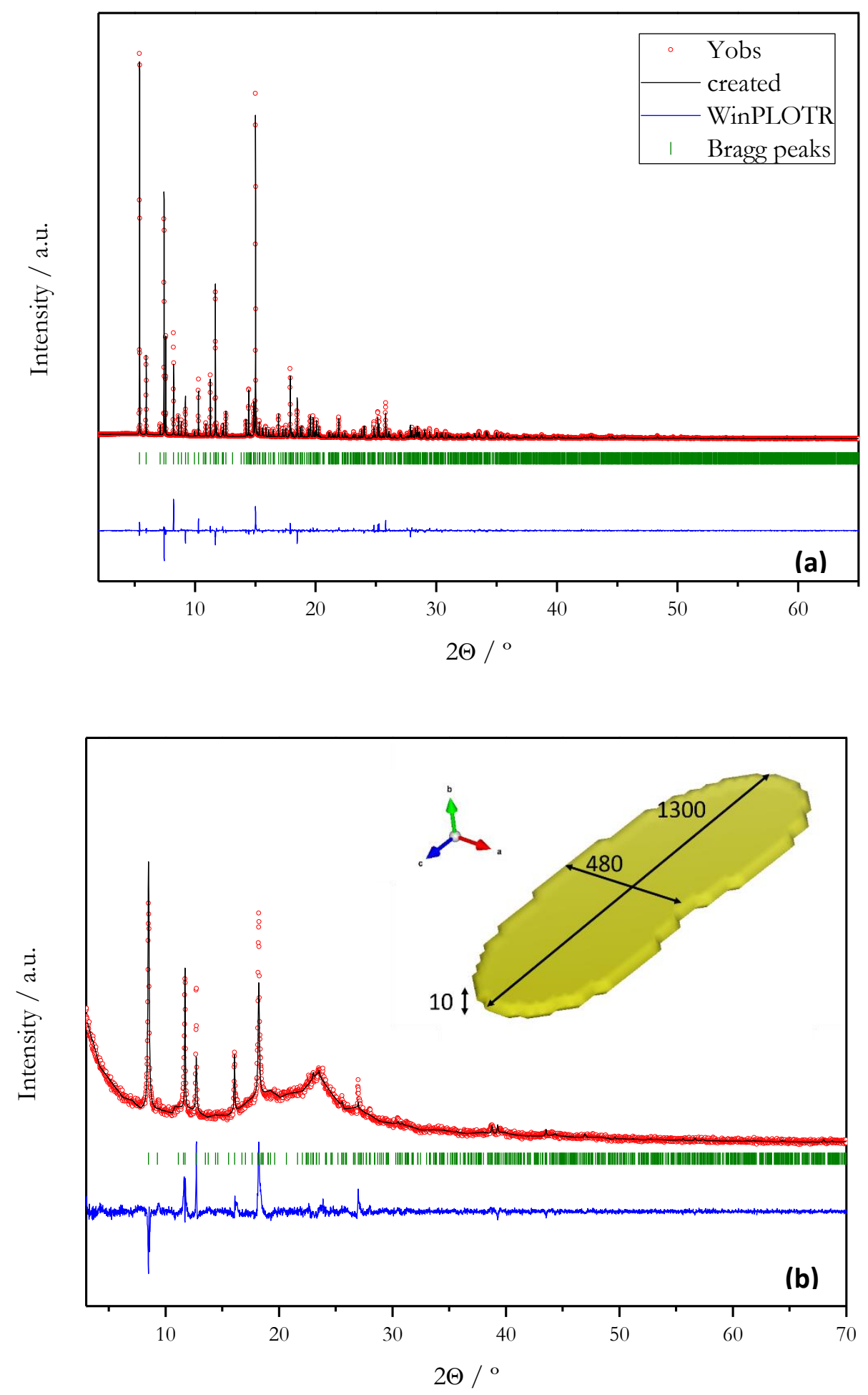

b)

Figure S2. Rietveld refinement at room temperature for the adduct obtained by slow evaporation (a) and by SAS at $313 \mathrm{~K}$ and $10 \mathrm{MPa}$ using ethanol as solvent (b), showing in the last case the average crystal domain sizes (in $\AA$ ) obtained by microstructural analysis. 
Table S1. Crystallographic data. Experimental parameters and structure refinement data for $\mathbf{C u}_{2}(\mathbf{C 3})_{4} \mathbf{I N A} \mathbf{A}_{4}$ obtained by slow evaporation and SAS.

\begin{tabular}{|c|c|c|}
\hline Crystal data & \multicolumn{2}{|c|}{$\mathrm{Cu}_{2}(\mathrm{C3})_{4} \mathrm{INA}_{4}$} \\
\hline Preparation method & Slow evaporation & SAS \\
\hline$M r\left(\mathrm{~g} \cdot \mathrm{mol}^{-1}\right)$ & 453.94 & 453.94 \\
\hline cell setting & monoclinic & monoclinic \\
\hline space group (no.) & $P 2_{1} / c(14)$ & $P 2_{1} / c(14)$ \\
\hline temperature $(\mathrm{K})$ & $298(2)$ & $298(2)$ \\
\hline$a(\AA)$ & $10.82731(7)$ & $10.7161(4)$ \\
\hline$b(\AA)$ & $12.38686(5)$ & $12.9(6)$ \\
\hline$c(\AA)$ & $15.48057(9)$ & $15.5614(6)$ \\
\hline$\beta\left({ }^{\circ}\right)$ & $103.4426(4)$ & $104.000(4)$ \\
\hline$V\left(\AA^{3}\right)$ & 2019.317(19) & 2086(101) \\
\hline $\mathrm{Z}, \mathrm{D}_{\mathrm{c}}\left(\mathrm{g} \cdot \mathrm{cm}^{-3}\right)$ & $4,1.493$ & $4,1.515$ \\
\hline wavelength $(\AA)$ & $0.9940(1)$ & $\mathrm{Cu} \mathrm{K \alpha 1}$ \\
\hline \multicolumn{3}{|l|}{ Data collection } \\
\hline diffractometer & $\begin{array}{l}\text { I711 (MAX IV } \\
\text { Laboratory) }\end{array}$ & $\begin{array}{c}\text { PANalytical } \\
\text { X'Pert MPD } \\
\text { (U.Complutense) }\end{array}$ \\
\hline specimen mounting & \multicolumn{2}{|c|}{ borosilicate glass capillary } \\
\hline data collection mode & \multicolumn{2}{|c|}{ transmission } \\
\hline scan mode & \multicolumn{2}{|c|}{$2 \theta$-step scan } \\
\hline $2 \theta$ range $\left(^{\circ}\right)$, step size $\left(^{\circ} 2 \theta\right)$ & $0-70,0.008$ & $5-60,0.016$ \\
\hline \multicolumn{3}{|l|}{ Refinement } \\
\hline Refinement method & \multicolumn{2}{|c|}{ full-matrix least-squares on $I_{\text {net }}$} \\
\hline$R_{p}, R_{w p}, R_{e x p}$ & $\begin{array}{c}0.0180,0.0354, \\
0.0508\end{array}$ & $\begin{array}{c}0.0581,0.0844 \\
0.0471\end{array}$ \\
\hline$R_{F}, R_{B R A G G}$ & $0.0785,0.0975$ & $0.0834,0.0224$ \\
\hline goodness-of-fit & 0.64 & 1.79 \\
\hline profile function & \multicolumn{2}{|c|}{$\begin{array}{l}\text { pseudo-Voigt with axial divergence } \\
\text { asymmetry }\end{array}$} \\
\hline no. contributing reflections & 3863 & 854 \\
\hline no. Parameters & 253 & 7 \\
\hline no. restraints & 149 & 3 rigid bodies \\
\hline CCDC deposition number & 1848049 & \\
\hline
\end{tabular}

The diffraction pattern was analyzed by Rietveld refinement using the Fullprof Suite 1-2 ${ }^{1,2}$ to evaluate the crystal size (from the broadening of the peaks in the whole pattern). The instrumental resolution function was obtained from $\mathrm{LaB}_{6}$ and all the patterns were fit with the Thompson-Cox-Hastings pseudo-Voigt profile function through the FullProf program.

The microstructure analysis shows an average crystal domain corresponding to a platelet, which is large in the directions (100) and (001) but extremely narrow in the 
direction (010); in the order of $10 \AA$. This direction corresponds to the stacking of the isonicotinamide aromatic rings. No sign of other crystalline phases (reactants, or new phases) was observed in this pattern.

(1) Rodríguez-Carvajal, J., Recent advances in magnetic structure determination by neutron powder diffraction. Phys. B. 1993, 192, 55-69.

(2) Rodríguez-Carvajal, J., Recent developments of the program FULLPROF. Commission on powder diffraction (IUCr). Newsletter 2001, 26, 12-19. 

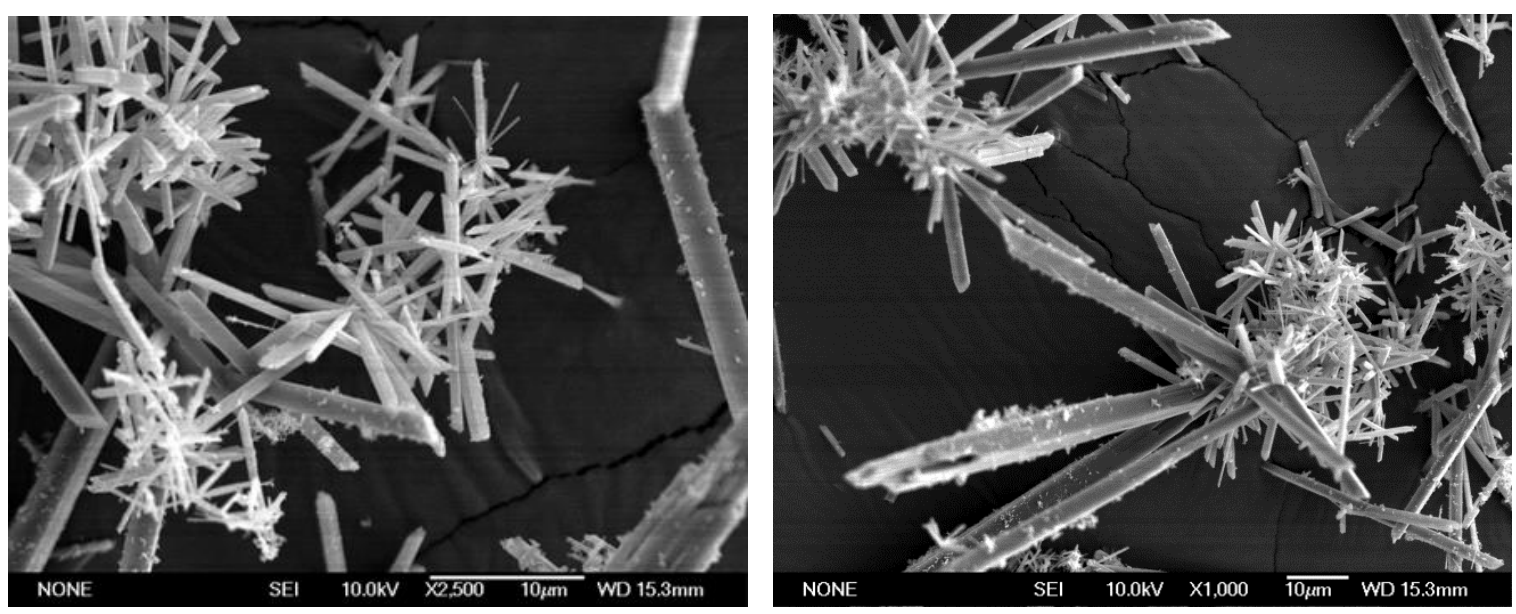

Figure S3. SEM images of the sample prepared by SAS at $313 \mathrm{~K}$ and $10 \mathrm{MPa}$ using methanol as a solvent. 
Table S2. Microelemental analysis of the samples prepared by evaporation and SAS at the different conditions.

\begin{tabular}{|c|c|c|c|c|}
\hline Adduct & $\begin{array}{c}\% \mathrm{C} \\
\pm \mathbf{0 . 3 5} \\
\end{array}$ & $\begin{array}{c}\% \mathrm{H} \\
\pm 0.30 \\
\end{array}$ & $\begin{array}{c}\% \mathrm{~N} \\
\pm 0.30 \\
\end{array}$ & $\begin{array}{l}\text { C:N } \\
\pm 0.35 \\
\end{array}$ \\
\hline $\mathrm{Cu}_{2}\left(\mathrm{C}_{3}\right)_{4}(\mathrm{INA})_{4}$ Theoretical & 47.63 & 4.88 & 12.34 & 4.50 \\
\hline Evaporation & 47.45 & 4.85 & 12.22 & 4.53 \\
\hline SAS at $313 \mathrm{~K}$ and $10 \mathrm{MPa} \mathrm{EtOH}-\mathrm{Cu}\left(\mathrm{C}_{3}\right)_{2}$ :INA $1: 2$ & 43.75 & 4.26 & 13.17 & 3.88 \\
\hline SAS at $298 \mathrm{~K}$ and $8 \mathrm{MPa}$ EtOH $-\mathrm{Cu}\left(\mathrm{C}_{3}\right)_{2}: \mathrm{INA} \quad 1: 2$ & 43.19 & 4.37 & 12.29 & 4.10 \\
\hline SAS at $313 \mathrm{~K}$ and $10 \mathrm{MPa} \mathrm{MeOH}-\mathrm{Cu}\left(\mathrm{C}_{3}\right)_{2}$ :INA $1: 2$ & 43.30 & 4.16 & 12.61 & 4.01 \\
\hline SAS at $298 \mathrm{~K}$ and $10 \mathrm{MPa} \mathrm{EtOH}-\mathrm{Cu}\left(\mathrm{C}_{3}\right)_{2}$ :INA $1: 2$ & 32.11 & 3.47 & 10.58 & 3.54 \\
\hline SAS at $313 \mathrm{~K}$ and $25 \mathrm{MPa} \mathrm{EtOH}-\mathrm{Cu}\left(\mathrm{C}_{3}\right)_{2}$ :INA $1: 2$ & 30.79 & 3.59 & 9.24 & 3.89 \\
\hline SAS at $313 \mathrm{~K}$ and $10 \mathrm{MPa}$ EtOH $-\mathrm{Cu}\left(\mathrm{C}_{3}\right)_{2}$ :INA $1: 1$ & 26.47 & 3.35 & 7.88 & 3.92 \\
\hline
\end{tabular}

